\author{
O R I G I N A L A R T I C L E
}

\title{
Dynamic solute release from marine aggregates
}

\author{
Bo Liu, ${ }^{1}$ Kolja Kindler, ${ }^{1}$ and Arzhang Khalilii, ${ }^{1,2}$
}

\begin{abstract}
Rapidly sinking marine snow aggregates play an important role in the vertical transport of carbon in the ocean. During their descent, particulate organic carbon is gradually turned over by microbial respiration and solubilization, while solutes are constantly released into the water. This steady-state scenario is interrupted when the aggregate passes through density discontinuities (pycnoclines), resulting in a retention period before resuming their decent. The accumulated excess solutes mediated by the absence of advection will then release dynamically into ambient water. In an attempt to quantify this dynamic process, we present numerical simulations of the flow adjacent to and solute release from porous spheres in a hydrodynamic regime representative of marine snow. We focus on the interdependence of internal diffusion-dominated transport and advective flow around an aggregate. The aggregate has been assumed to have a constant size, excess density, and permeability. We propose a new relationship for the dynamic release of solutes from sinking, porous aggregates in terms of the average Sherwood number, $\overline{\mathrm{Sh}}=1+8 \mathrm{Pe}^{0.6} /\left(17.4+\mathrm{Pe}^{0.6}\right)$, as a function of the Peclét number, $\mathrm{Pe}$, where $\mathrm{Pe}=\mathrm{aw}_{\mathrm{s}} \mathrm{D}^{-1}$, a is the radius of the sphere, $\mathrm{w}_{\mathrm{s}}$ is the settling velocity, and $\mathrm{D}$ is the diffusion coefficient. An additional degradation rate constant, which was considered, did not change this finding significantly. From this relationship, release times and export depths-that is, the distances within which 99\% of the excess solute is released into the ambient water-can be readily obtained as a function of aggregate size and excess density.
\end{abstract}

Keywords: marine snow, solute leakage, porous sphere, Sherwood number, lattice Boltzmann method

${ }^{1}$ Max Planck Institute for Marine Microbiology, Bremen, Germany

${ }^{2}$ Department of Earth and Environmental Sciences, Jacobs University Bremen, Bremen, Germany

Correspondence to

Bo Liu,

bliu@mpi-bremen.de

\section{Introduction}

[1] The settling of marine aggregates in the water column has significant effects on the biogeochemistry and ecology of the oceans (Asper et al. 1992). Owing to their relatively high sinking speeds, aggregates might be thought of as vehicles for the vertical flux of organic matter in aquatic environments. Marine aggregates also constitute hot spots of microbial activity and are sites of rapid and efficient turnover of particulate organic carbon in the ocean (Alldredge and Silver 1988; Simon et al. 2002).
[2] Marine aggregates appear in many different forms with varying hydrodynamic properties (Turner 2002). Larger-scale aggregates, commonly referred to as marine snow, have radii, $a$, of $0.5-10 \mathrm{~mm}$ (e.g., Alldredge and Gotschalk 1990), with settling speeds, $w_{s}$, ranging from $O\left(10 \mathrm{~m} \mathrm{~d}^{-1}\right)$ to $O\left(100 \mathrm{~m} \mathrm{~d}^{-1}\right)$, where $O$ is an order of magnitude. The latter type of aggregates is highly porous, with porosities, $\varepsilon$, frequently exceeding $90 \%$, depending on type and composition (Leppard et al. 2004; Turner 2002). Permeability, $k$, within aggregates 
has been found to range from $O\left(10^{-15} \mathrm{~m}^{2}\right)$ for marine snow to $O\left(10^{-9} \mathrm{~m}^{2}\right)$ for sludge flocs (Gorczyca and Ganczarczyk 2002; Li et al. 2003; Ploug and Passow 2007). Aggregates have also been found to be fractallike when formed by sequential coagulation ( $\mathrm{Li}$ and Logan 2001).

[3] During the descent of an aggregate, the flux of dissolved organic carbon (DOC), nitrate, ammonium, and so forth, from within an aggregate into the ambient water is balanced by microbial respiration and solubilization within the aggregate. The internal concentration of solutes depends on the rate of transport from the aggregate; hence, this rate governs the rate of concentration-dependent processes. The steady exchange of solutes between the aggregate and the ambient water is dependent on flow, reaction rate, and molecular diffusion. However, when the flow or reaction undergoes a rapid change, this steady-state scenario is interrupted.

[4] When an aggregate sinks from rest suddenly, the internal concentration of solutes decreases due to the increased flux from the aggregate into the ambient water as a result of advection. In contrast, the internal concentration of solutes increases when a sinking aggregate suddenly comes to rest. Both of these dynamic processes of solute exchange occur at density discontinuities (pycnoclines), where aggregates are retained and form thin layers in the ocean (MacIntyre et al. 1995). This process has been demonstrated in laboratory experiments on real aggregates ( $\mathrm{Li}$ et al. 2003) and porous hydrogel spheres (Kindler et al. 2010), where typical marine aggregates were found to have retention times of many days at pycnoclines (Li et al. 2003). During this time, the dissolved organic matter inside aggregates increases due to the lack of advection. The excess dissolved organic matter will be released when the aggregate resumes descent and can either migrate back into the pycnocline or be transported with the aggregate to deeper layers. This process has implications for the "biological pump" in terms of additional respiration (release of $\left.\mathrm{CO}_{2}\right)$ and for the regeneration of primary production.

[5] A rapid modification of the reaction conditions can also change the internal solute concentration. For example, a reduction in light conditions at night can lead to the release of excess oxygen accumu- lated from photosynthesis during the day (Ploug et al. 1999, 2011).

[6] Hitherto, the underlying exchange processes involving marine aggregates have mostly been approximated by simple, steady-state model representations (e.g., solid sphere of radius $a$ sinking with a constant velocity $w_{s}$; Clift et al. 1978; Karp-Boss et al. 1996; Kiørboe et al. 2001), where mass transfer at the surface of solid spheres is governed by the concurrence of external advection and diffusion. The relative fluid motion induced by sinking steepens the surrounding solute gradients and thus increases the flux of a given solute leaving the particle surface. The nature of the flow is determined by the ratio of inertial to viscous force described by the Reynolds number,

$$
R e=a w_{s} \nu^{-1},
$$

where $\nu$ is the kinematic viscosity. The enhanced mass transfer determined by the ratio of the total flux of a solute leaving the surface of the moving cell $(J)$ to the diffusive flux of a stationary cell $\left(J_{D}\right)$ is described by the Sherwood number, Sh. For creeping flow $(R e<0.1)$,

$$
S h=0.5\left(1+(1+2 P e)^{0.333}\right),
$$

where $P e$ is the Peclét number given by

$$
P e=a w_{s} D^{-1},
$$

where $D$ is the diffusion coefficient (Clift et al. 1978). Kiørboe et al. (2001) proposed that

$$
S h=1+0.619 R e^{0.412} S c^{0.333}
$$

for $0.1<R e<20$, where $S c$ is the Schmidt number given by

$$
S c=\nu D^{-1} .
$$

[7] There have been several analytical and numerical studies of creeping flow through porous permeable spheres (Adler 1981; Rogak and Flagan 1990; Vanni 2000). Adler (1981) calculated the streamlines around and within a sinking porous sphere by applying the Brinkman extension of Darcy's law to a porous medium and the Stokes equation to the external creeping flow (see Appendix for Darcy's law and Brinkman's extension). Nandakumar and Masliyah (1982) computed the flow past a permeable sphere for intermediate Re by replacing the Stokes equation with the Navier-Stokes 
equation for external flow. Rogak and Flagan (1990) studied the creeping flow around self-similar clusters of spheres, and Vanni (2000) investigated creeping flow past a fractal aggregate with radially varying solid fractions and permeabilities.

[8] The present study focuses on solute release from a sinking aggregate in the vicinity of a pycnocline. It investigates the solute release mechanism as a dynamic process using a porous sphere, in which solute release is directly linked to the flow in and around the aggregate.

\section{Methods}

\section{Problem Statement}

[9] We consider a sphere of radius $a$ with uniform porosity $\varepsilon$ and permeability $k$ (Fig. 1 ). The porosity is defined as the fraction of the total volume that is occupied by void space. The permeability can be determined from the aggregate porosity with a Davies correlation,

$$
\frac{1}{k}=\frac{16}{a_{\mathrm{cyl}}^{2}}(1-\varepsilon)^{1.5}\left[1+56(1-\varepsilon)^{3}\right],
$$

where $a_{\text {cyl }}$ is the radius of a thin cylinder or filament composing an aggregate (Masliyah and Polikar 1980; Logan and Hunt 1987). The porous spheres are held fixed and subjected to a stationary, constant upward flow at far-field velocity $\left(w_{s}\right)$. At time $t=0$, the aggregate is saturated with a maximum concentration $\left(c_{0}\right)$, which was released into the ambient fluid due to the combined effect of internal diffusion and external diffusion and advection. The far-field concentration of the tracer solute within the ambient water was set to zero.

[10] The most important parameters affecting the settling velocity of aggregates are their size $(0.5 \mathrm{~mm} \leq a$ $\leq 10 \mathrm{~mm})$ and excess density $(\Delta \hat{\rho})$, defined by

$$
\Delta \hat{\rho}=\frac{(1-\varepsilon)\left(\rho_{s}-\rho\right)}{\rho},
$$

with $\rho_{s}$ as the density of the solid content. Excess density is of particular interest and might vary over orders of magnitude depending on aggregate composition, with typical values in the range of $\Delta \hat{\rho}=O\left(10^{-5}\right)$ to $\Delta \hat{\rho}=O\left(10^{-4}\right)$ (e.g., Alldredge and Silver 1988 Aggregates containing calcite disks from coccolithophorids or ballasting minerals might be in the range $\Delta \hat{\rho}=O\left(10^{-3}\right)$
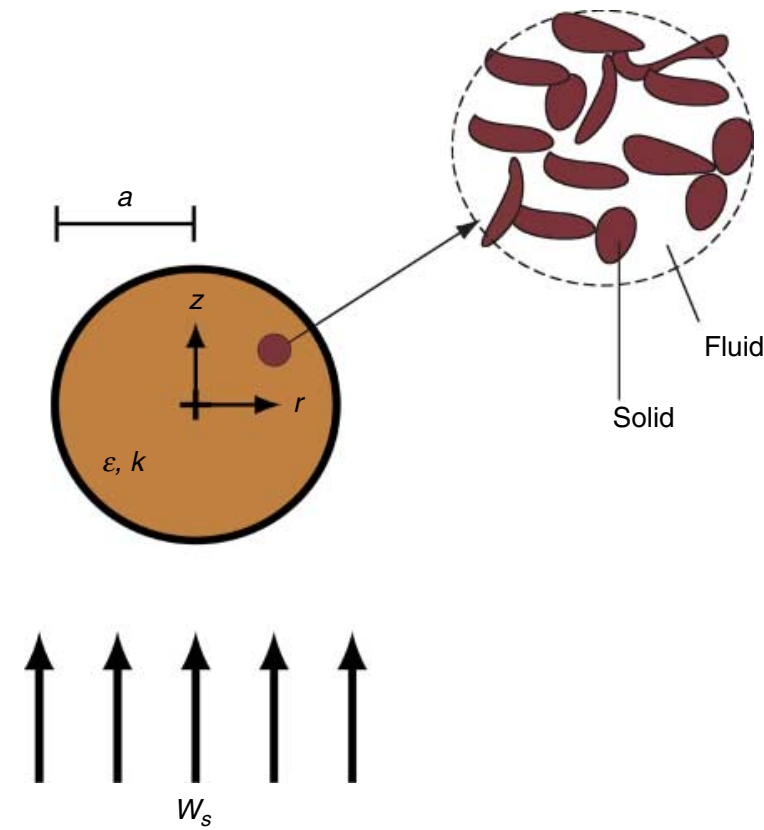

Fig. 1 Stagnant sphere of radius $a$, porosity $\varepsilon$, and permeability $k$ subjected to stationary flow (far-field settling velocity $w_{s}$ ), representing steady settling. At time $t=0$, the porous sphere contains a uniformly distributed solute concentration $c_{0}$, while the far-field concentration is set to zero.

to $\Delta \hat{\rho}=O\left(10^{-2}\right)$ (Iversen and Ploug 2010). For this reason, we analyze the range of $10^{-5}<\Delta \hat{\rho}<10^{-3}$.

[11] To ensure the hydrodynamic similarity with natural sedimentation in the ocean, $0.01 \leq R e \leq 10$ was chosen as characteristic of marine snow. An $S c=700$ has been used to represent tracer solutes such as DOC, amino acids, and dissolved gases (Kiørboe et al. 2001; Ploug and Passow 2007). The resulting Peclét number is in the range of $7 \leq P e \leq 7000$ (advection-dominated external flow). The permeability can be nondimensionalized using the square of the aggregate's radius, leading to the Darcy number, $D a$, given by

$$
D a=\frac{k}{a^{2}},
$$

which ranges from $10^{-11}$ to $10^{-3}$ in the present study.

\section{Basic Equations}

[12] Low $R e$ flow inside a porous aggregate can be described by Darcy's law including Brinkmann's extension (Basu and Khalili 1999; Guo and Zhao 2002). With this, the momentum and the concentration equations are 

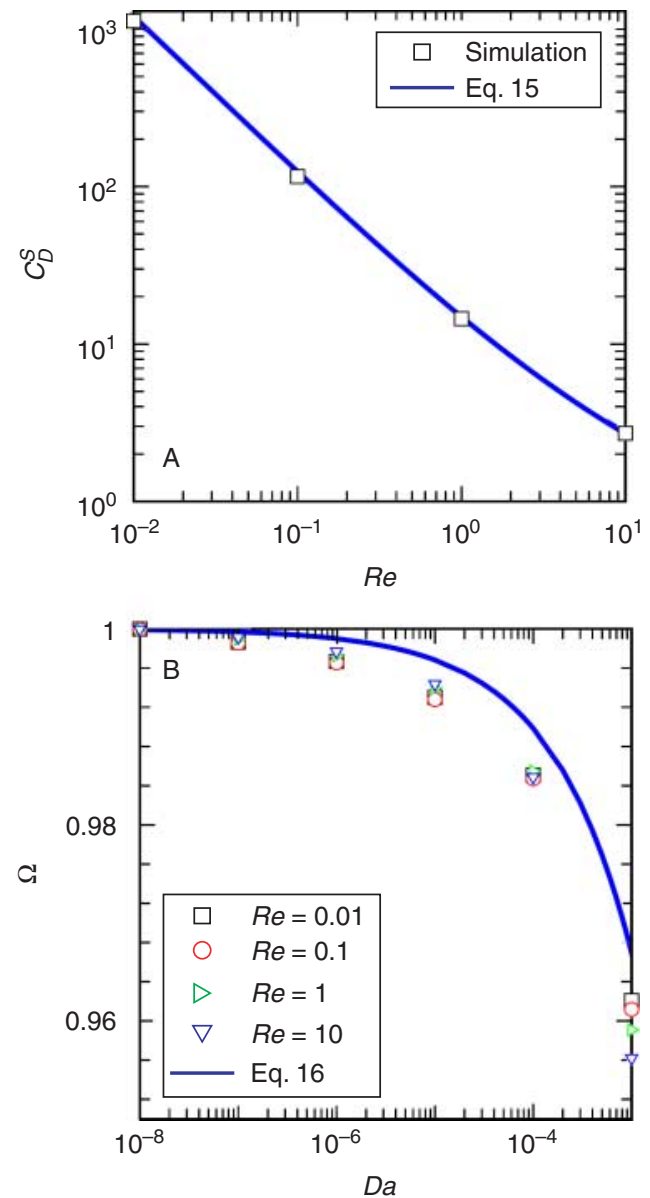

Fig. 2 A - Reynolds number (Re) dependence of the drag coefficient $C_{D}^{S}$ of solid spheres obtained from numerical simulation, compared with the empirical relationship of White (2005). B - Darcy number (Da) dependence of the drag ratio $\Omega$ of permeable spheres.

given by

$$
\begin{gathered}
\frac{\partial \mathbf{u}}{\partial t}+\nabla \cdot\left(\frac{\mathbf{u u}}{\varepsilon}\right)=-\frac{1}{\rho} \nabla(\varepsilon p)+\nu \nabla^{2} \mathbf{u}-A \frac{\varepsilon \nu}{k} \mathbf{u}, \\
\varepsilon \frac{\partial c}{\partial t}+(\mathbf{u} \cdot \nabla) c=\nabla \cdot(D \varepsilon \nabla c),
\end{gathered}
$$

where $\mathbf{u}, t, \rho, p, c$, and $A$ denote velocity vector, time, density, pressure, concentration, and a binary constant discriminating the fluid phase $(A=0)$ from the porous matrix $(A=1)$. According to this formulation, the fluid phase is defined by $\varepsilon=1, A=0$, which is equivalent to the Navier-Stokes equation for the motion of viscous fluid. Likewise, the porous region occupied by the aggregate dynamically is given by $\varepsilon=0.95, A=1$, which is the extension of the Darcy equation. Compared with the Darcy equation, which is the balance of the pressure gradient $(\nabla p)$ and Darcy force $\left(\frac{\nu}{k} \mathbf{u}\right)$, Eq. 9 takes into account the change of velocity with time $\left(\frac{\partial \mathbf{u}}{\partial t}\right)$, the advection $\left[\nabla \cdot\left(\frac{\mathbf{u u}}{\varepsilon}\right)\right]$, and the viscous force known as the Brinkman term $\left(\nu \nabla^{2} \mathbf{u}\right)$. The boundary condition across the interface will automatically satisfy the continuity in velocity and concentration as well as conservation of mass (i.e., chemical flux across the surface is continuous). For more details of application of this equation to porous media, see Nield and Bejan (1998).

[13] Because of the symmetry of the geometry and flow with respect to the $z$-axis, we made use of axis symmetry, leading to lower computational time. The above equations, with the addition of the continuity equation (with $r$ as the cylinder radius and $z$-axis direction),

$$
\frac{\partial w}{\partial z}+\frac{1}{r} \frac{\partial(r u)}{\partial r}=0
$$

can be rewritten in nondimensional form, using cylindrical coordinates

$$
\begin{gathered}
\frac{\partial w}{\partial t}+\frac{1}{\varepsilon} \frac{\partial\left(w^{2}\right)}{\partial z}+\frac{1}{r \varepsilon} \frac{\partial(r w u)}{\partial r}=-\frac{\partial(\varepsilon p)}{\partial z}-\frac{\varepsilon w}{R e D a} \\
+\frac{1}{R e}\left[\frac{\partial^{2} w}{\partial z^{2}}+\frac{1}{r} \frac{\partial}{\partial r}\left(r \frac{\partial w}{\partial r}\right)\right],
\end{gathered}
$$

$$
\begin{aligned}
\frac{\partial u}{\partial t}+\frac{1}{\varepsilon} \frac{\partial(w u)}{\partial z}+\frac{1}{r \varepsilon} \frac{\partial\left(r w^{2}\right)}{\partial r}= & -\frac{\partial(\varepsilon p)}{\partial r}-\frac{\varepsilon u}{R e D a} \\
& +\frac{1}{R e}\left[\frac{\partial^{2} u}{\partial z^{2}}+\frac{1}{r} \frac{\partial}{\partial r}\left(r \frac{\partial u}{\partial r}\right)-\frac{u}{r^{2}}\right]
\end{aligned}
$$

$$
\frac{\partial c}{\partial t}+\frac{w}{\varepsilon} \frac{\partial c}{\partial z}+\frac{u}{\varepsilon} \frac{\partial c}{\partial r}=-\frac{1}{R e S c}\left[\frac{\partial^{2} c}{\partial z^{2}}+\frac{1}{r} \frac{\partial}{\partial r}\left(r \frac{\partial c}{\partial r}\right)\right]
$$

where length, time, density, pressure, and concentration are scaled by $a, \nu a^{-2}, \rho, \rho w_{s}^{2}$, and $c_{0}$, respectively, while $u$ and $w$ are scaled velocities in the $r$ and $z$ directions, respectively. The initial concentration of solute inside the aggregate and in the fluid domain is given by $c=1$ and $c=0$, respectively.

\section{Numerical Solution Method}

[14] To solve Eqs. 11-14, we have implemented the lattice-Boltzmann method (LBM). The root of LBM 

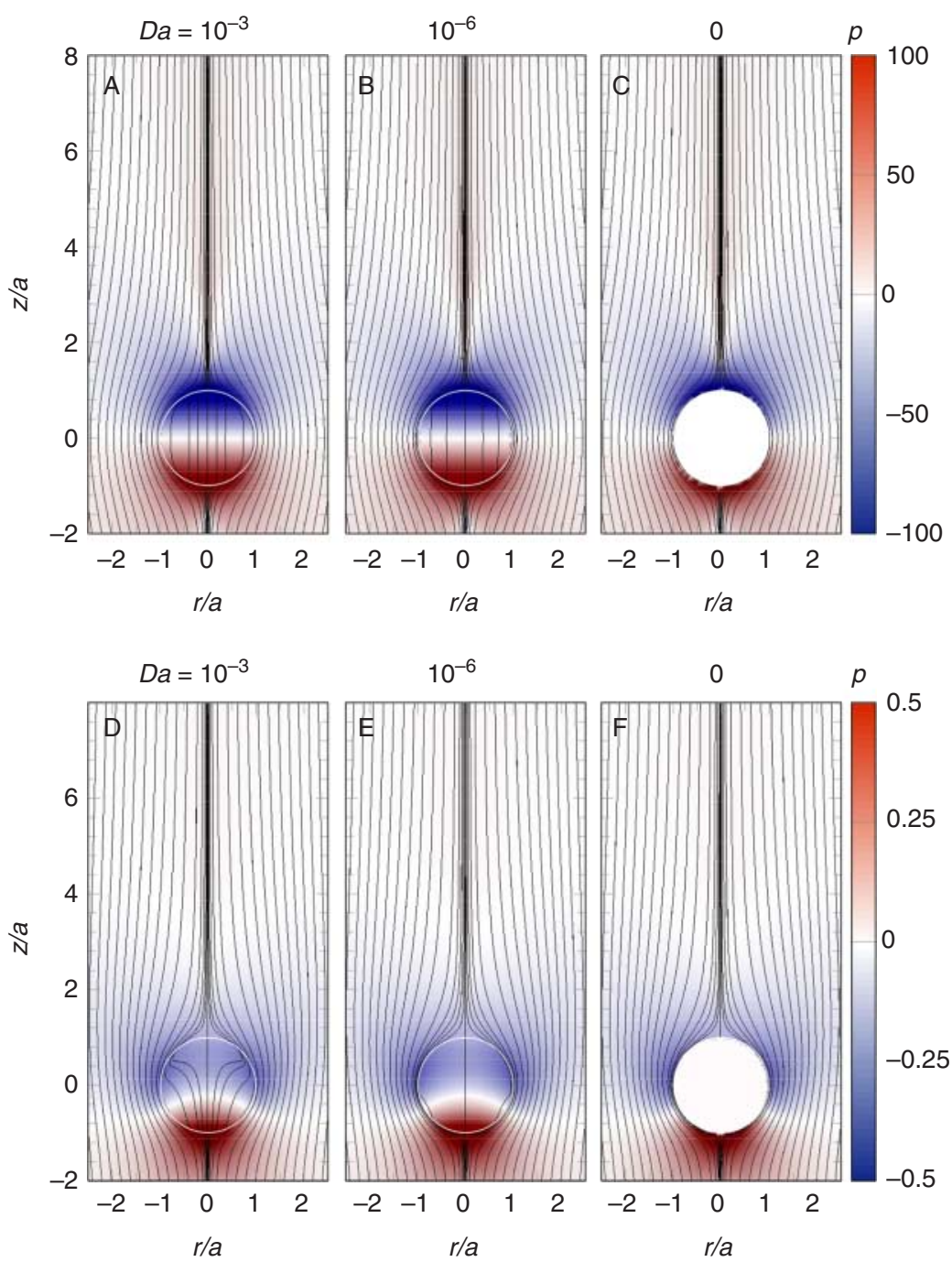

Fig. 3 Pressure fields and Stokes streamlines for porous spheres of varying Darcy numbers, $D a$, settling at $\operatorname{Re}=0.01(\mathrm{~A}-\mathrm{C})$ and $R e=10(\mathrm{D}-\mathrm{F})$.

lies in kinetic theory and was developed initially to simulate complex fluid flow. LBM has become very popular in last two decades, mainly because of its simplicity and its scalability on parallel processing systems. Furthermore, with LBM it is easy to describe microscopic interactions in multicomponent/multiphase systems. It is applicable for complex fluid systems without suitable governing equations (Succi 2001).

[15] Whereas conventional numerical schemes are based on macroscopic continuum equations, LBM is based on microscopic and mesoscopic kinetic equations. The principal idea of the LBM is to construct simplified kinetic models that incorporate the essential physics of microscopic or mesoscopic processes so that the macroscopic averaged properties obey the desired macroscopic equations (Wolf-Gladrow 1998; Chen and Doolen 1998; Aidun and Clausen 2010). The axisymmetric LBM implemented here was first introduced by Guo et al. (2009) and has been explained in detail in the appendix for the sake of completeness.

\section{Validation}

[16] The numerical results for the hydrodynamics were validated in two steps. First, the drag coefficients of solid (nonpermeable) spheres, $C_{D}^{S}$, were found to be in excellent agreement with the empirical relationship (White 2005)

$$
C_{D}^{S}=\frac{12}{R e}+\frac{6}{1+\sqrt{2 R e}}+0.4
$$

in the $R e$ regime relevant to this work (Fig. 2A). Second, the impact of permeability on the drag exerted on the porous sphere was investigated. The ratio of drag coefficient for a permeable sphere, $C_{D}^{P}$, to that of a solid one is expressed as $\Omega=C_{D}^{P} / C_{D}^{S}$ and was derived (Neale et al. 1973) for the creeping flow regime as

$$
\Omega=\frac{2 D a^{-1}\left[1-\tanh \left(D a^{-0.5}\right) / D a^{-0.5}\right]}{2 D a^{-1}+3\left[1-\tanh \left(D a^{-0.5}\right) / D a^{-0.5}\right]} .
$$

Comparing our numerical results with those of Eq. 16, negligible deviations of $<1 \%$ were found for $D a \leq 10^{-3}$ and $R e \leq 10$ (Fig. $2 \mathrm{~B}$ ). This provides the justification in our model, in which the permeability has been considered to be uniform throughout the aggregate.

\section{Results}

Velocity and Pressure Fields

[17] In the Stokes regime $(R e<0.1)$, flow fields were symmetric (Fig. 3), but increasing Re results in diminishing front-aft symmetry, because of the increasing im- 

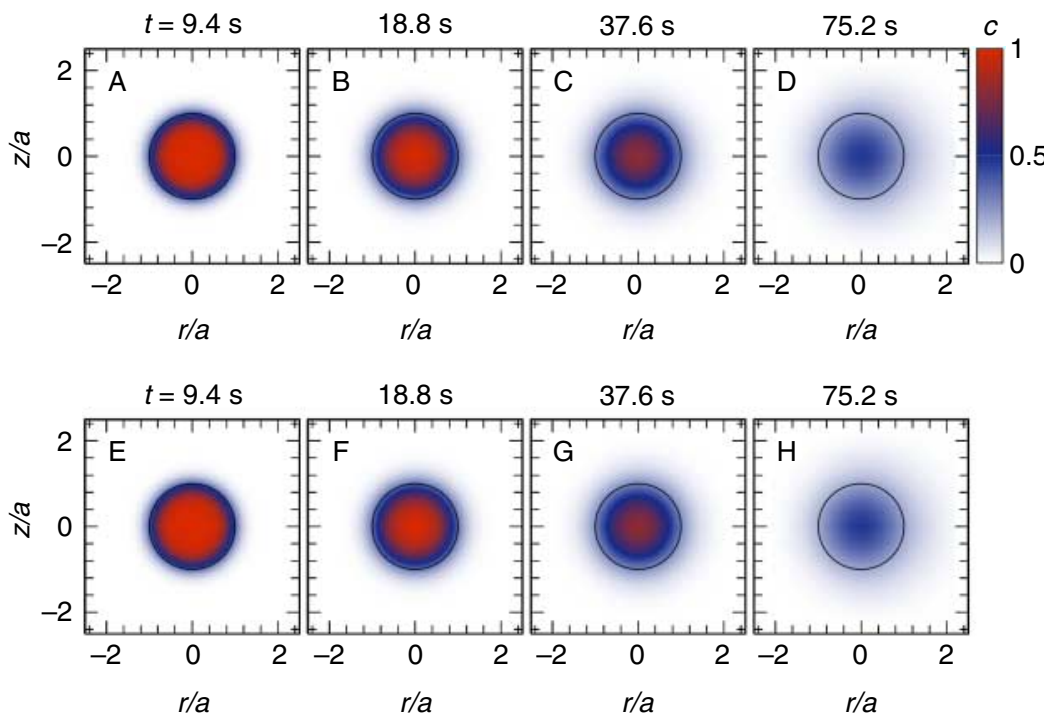

Fig. 4 Time series of diffusion-dominated solute release from a porous sphere (radius $a=0.45 \mathrm{~mm}$ ) with $w_{s}=1.9 \mathrm{~m} \mathrm{~d}^{-1}(\operatorname{Re}=0.01), D=1.4 \times 10^{-9} \mathrm{~m}^{2} \mathrm{~s}^{-1}(\mathrm{Sc}=700)$, and $\Delta \hat{\rho}=5 \times 10^{-5}$ (saturated concentration $c_{0}=1$ at $t=0$ ). A-D - Solute release from an aggregate with high permeability, $k=2.0 \times 10^{-10} \mathrm{~m}^{2}\left(\mathrm{Da}=10^{-3}\right) . \mathrm{E}-\mathrm{H}-$ Solute release from an aggregate with weak permeability, $k=2.0 \times 10^{-13} \mathrm{~m}^{2}\left(\mathrm{Da}=10^{-6}\right)$.

pact of inertial forces in the wake. Flow fields around porous spheres for very low permeabilities were similar to those of solid spheres (Fig. 3), in agreement with results for the drag coefficients reported above.

[18] To highlight the differences, two extreme Reynolds numbers $(R e=0.01$ and $R e=10)$ were considered for high $\left(D a=10^{-3}\right)$, weak $\left(D a=10^{-6}\right)$, and null permeability (solid sphere) (Fig. 3). Several distinct features of the flow can be discerned. At a low Re and high $D a$, flow fields within the aggregates were almost symmetric upstream and downstream (Fig. 3A). With increasing $R e$, the streamlines deformed because of a change of pressure profile (Fig. 3D). This effect was also reflected in an almost horizontal, interstitial pressure interface that increasingly bulges outward at larger $R e$ values.

\section{Solute Transfer}

[19] In order to provide real-time estimates of solute release, we fixed the excess density $\Delta \hat{\rho}=5 \times 10^{-5}$. A time series of images of the concentration field of highly ( $D a=10^{-3}$, corresponding to the permeability $\left.k=2.0 \times 10^{-10} \mathrm{~m}^{2}\right)$ and weakly $\left(D a=10^{-6}\right.$, corresponding to $k=2.0 \times 10^{-13} \mathrm{~m}^{2}$ ) permeable aggregates of small size $(a=0.45 \mathrm{~mm})$ revealed that slow settling motions $(R e=0.01$, $w_{s}=1.9 \mathrm{~m} \mathrm{~d}^{-1}$ ) have a minor impact on solute exchange (Fig. 4). In the Stokes regime, the permeability is negligible, because permeation was weak enough for solute release to be driven mostly by diffusion. This was also reflected in a nearly symmetric concentration field. Conversely, the effect of permeability was visible for a large aggregate $(a=5.9 \mathrm{~mm})$ due to its high settling velocity $\left(R e=10, w_{s}=147 \mathrm{~m} \mathrm{~d}^{-1}\right)$. For an aggregate of high permeability $\left(D a=10^{-3}, k=3.5 \times 10^{-8} \mathrm{~m}^{2}\right)$, the effect of internal advection on the solute release was also evident (Fig. 5A-D). However, for an aggregate of small permeability $\left(\mathrm{Da}=10^{-6}, \quad k=3.5 \times 10^{-11}\right.$ $\mathrm{m}^{2}$ ), internal solute release takes place in a rather diffusive manner (Fig. $5 \mathrm{E}-\mathrm{H})$. In both cases, the solutes released into the fluid domain form plumes at the downstream side of the aggregate in the shape of a hollow cylindrical cone, which successively merge into a single slender tail.

[20] For practical purposes, fractional release of the initial amount of solute was calculated (Fig. 6) for four different $R e$ (corresponding to four different $a$ ) as a function of depth traveled $(z)$. Except for $R e=10$ and $D a=10^{-3}$, the effects of permeability can be neglected. Furthermore, in all cases the aggregates released their initial solute content within the upper 100-m depth. The coincidence of the dashed (weak-permeable $D a=10^{-6}$ ) and solid (high-permeable $D a=10^{-3}$ ) lines at $R e=0.01,0.1$, and 1 (Fig. 6A-C) provide a further confirmation of the calculations (Fig. 4). Moreover, the deviation of the data for $R e=10$ (Fig. 6D) is the consequence of the internal advection (Fig. 5).

\section{Discussion}

\section{Solute Release Enhancement}

[21] To characterize the solute release dynamics, we consider two release times $\left(T_{D}\right.$ and $\left.T\right)$, within which $99 \%$ of initial solute content was discharged. $T_{D}$ denotes the 

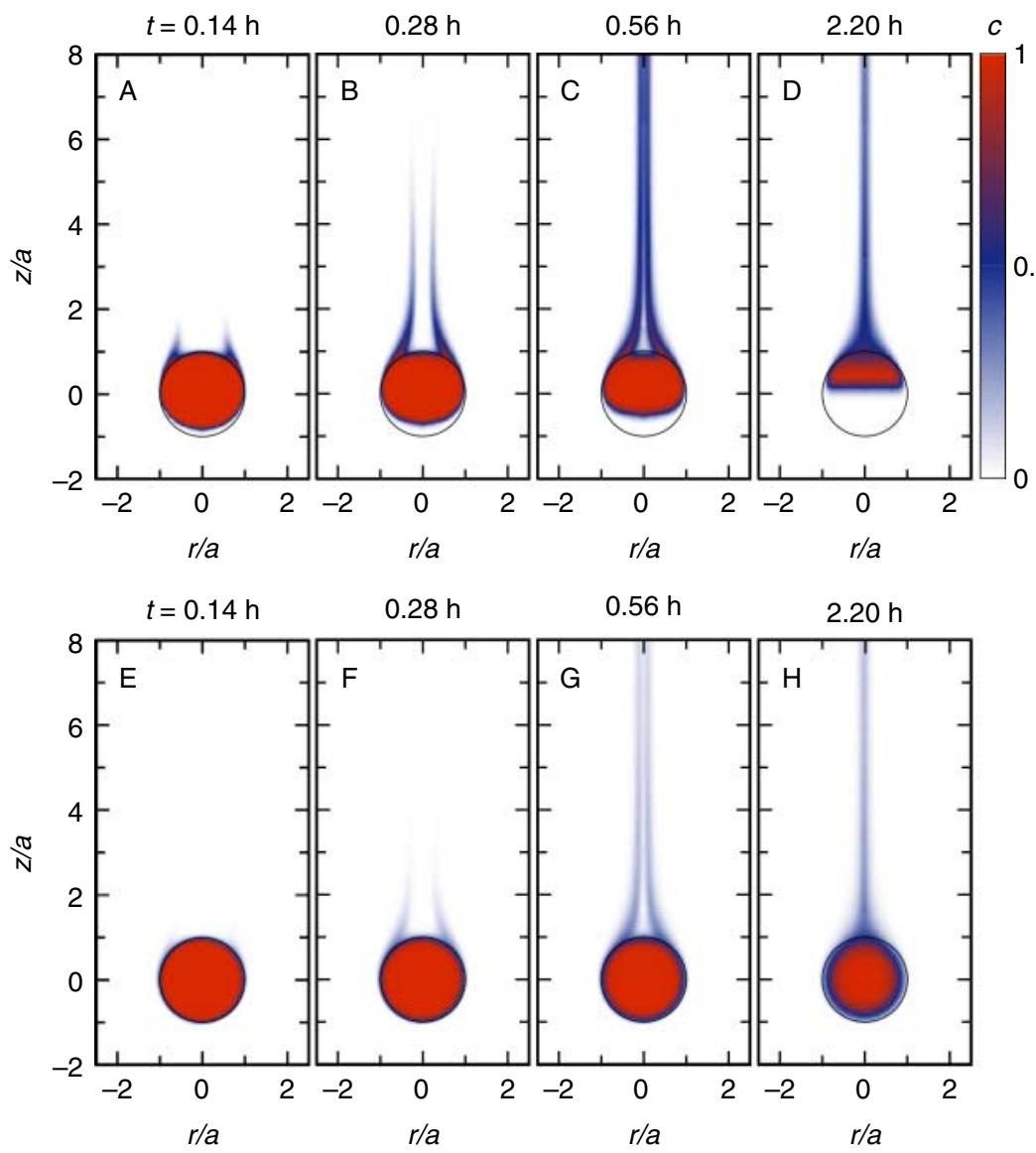

Fig. 5 Time series of advection-affected solute release from a porous sphere (radius $a=5.9 \mathrm{~mm}$ ) with $w_{s}=147 \mathrm{~m} \mathrm{~d}^{-1}(R e=10)$. A-D - Solute release from an aggregate with high permeability, $k=3.5$ $\times 10^{-8} \mathrm{~m}^{2}\left(\mathrm{Da}=10^{-3}\right) . \mathrm{E}-\mathrm{H}-$ Solute release from an aggregate with for weak permeability, $k=3.5$ $\times 10^{-11} \mathrm{~m}^{2}\left(D a=10^{-6}\right)$. All other parameters are as described for Fig. 4 .

release time associated with pure diffusion (stagnant aggregate), while $T$ denotes the release time associated with diffusion and advection (sinking aggregate). The averaged Sherwood number can then be defined by

$$
\overline{S h}=\frac{T_{D}}{T} .
$$

The purely diffusion-driven release time $T_{D}$ obtained from numerical simulations is $T_{D}=3.45 \times 10^{4} a^{2} D^{-1}$.

[22] We considered a porous sphere nearly impermeable to flow $\left(D a=10^{-8}\right)$, and the average solute mass transfer enhancement $\overline{S h}$ as a function of $P e$ was computed from the numerical results (solid line in Fig. 7). $\overline{S h}$ converged toward constant values at large $P e$ values. We fitted the $\overline{S h}$ data to the form $1+A P e^{B}\left(C+P e^{B}\right)^{-1}$, with
$A, B$, and $C$ as free parameters. The best fit over the entire $P e$ range $7 \leq P e \leq$ 7000 yields

$$
\overline{S h}=1+\frac{8 P e^{0.60}}{17.4+P e^{0.60}},
$$

which converges to

$$
\lim _{P e \rightarrow \infty} \overline{S h}=9 .
$$

The existence of this bound for $\overline{S h}$ can be attributed to both the diffusion-dominated transport within the sphere and the diminishment of the concentration boundary layer at sufficiently large $P e$ values. The finite limiting value of $\overline{S h}$ provides an estimate for the aggregate's fastest response to changing environmental conditions, where the lowest relaxation time of the interstitial microbial community is given by $T=T_{D} / 9=3.8 \times 10^{3} a^{2} D^{-1}$.

[23] The practical use and the significance of Eq. 18 are demonstrated below. The sinking speed of an aggregate can be derived from a force balance between the gravity and drag forces as

$$
\frac{1}{2} w_{s}^{2} \pi a^{2} C_{D}^{P}=\Delta \hat{\rho} \frac{4}{3} \pi a^{3} g
$$

leading to

$$
w_{s}=\sqrt{\frac{8 g}{3 C_{D}^{P}} \Delta \hat{\rho} a} .
$$

Combining Eqs. 1, 15, and 21 yields the settling velocity $w_{s}$ for a given $a$ and $\Delta \hat{\rho}$. With this $w_{s}, P e$ can be calculated from Eq. 3 to obtain $\overline{S h}$ from Eq. 18.

[24] To emphasize the difference between dynamic and steady-state solute release, the $S h-P e$ trend for a steady state was included in Fig. 7 (dashed and dotdashed lines). The averaged mass transfer of an aggregate with $a=1 \mathrm{~mm}, \Delta \hat{\rho}=10^{-4}$, and $w_{s}=17 \mathrm{~m} \mathrm{~d}^{-1}$ would be $\overline{S h}=5.19$, which is $36 \%$ above the value 


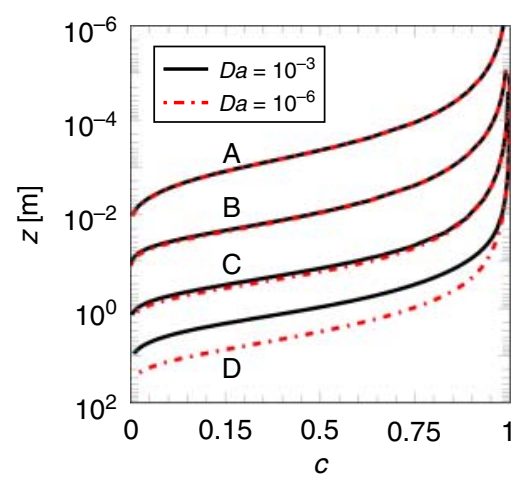

Fig. 6 Depth profiles of solute release at $R e=0.01(\mathrm{~A}), \operatorname{Re}=0.1(\mathrm{~B}), \operatorname{Re}=1(\mathrm{C})$, and $R e=10$ (D). The lines in the figure demonstrate the percentage of the solute (c) that remained in the aggregate at a given depth, with $c_{0}=1$ at $t=0$ and $z=0$. Note that the Darcy number effects were negligible for small Reynolds numbers; only at $R e=10$ was permeation noticeable.

obtained from Eq. 4 and 38\% larger than Eq. 2. Similarly, for an aggregate with $a=2.5 \mathrm{~mm}, \Delta \hat{\rho}=10^{-4}$, and $w_{s}=82 \mathrm{~m} \mathrm{~d}^{-1}$, the mass transfer obtained from Eq. 18 would amount to $\overline{S h}=7.64,13 \%$ below Eq. 4 and $4 \%$ less than Eq. 2. Hence, the combination of Eqs. 18 and 21 facilitates quantitative estimates of solute release for the whole spectrum of marine aggregates for given particle size distributions and known excess densities.

\section{Ballasting Effects}

[25] The impact of varying excess density on setting velocities and transfer rates as a function of aggregate radius is shown in Fig. 8. The settling velocity of porous aggregates as a function of aggregate size for different excess densities is shown in Fig. 8A. Note that for smaller radii, settling velocity is proportional to $a^{2}$ in accordance with the Stokes velocity

$$
w_{s}=\frac{2}{9 \nu} g \Delta \hat{\rho} a^{2} .
$$

[26] The average Sherwood number, $\overline{S h}$, as a function of size is depicted in Fig. $8 \mathrm{~B}$ and shows two different trends. For smaller radii, the averaged mass transfer from porous aggregates increases with an increase of excess density, whereas for larger radii, the limiting value of $\overline{S h} \approx 9$ is independent of excess density. From the $\overline{S h}$ values presented in Fig. $8 \mathrm{~B}$ and Eq. 17, one can arrive at the solute release time as a function of aggregate radii (Fig. 8C). At larger radii, the dynamic solute release time is $T \sim a^{2}$, indicating that mass transfer from the aggregate's interior is a diffusion-limited process at large $R e$, as explained above.

[27] For practical applications, it is useful to estimate the water depth $H$ at which $99 \%$ of the initially available nutrient at $z=0$ is released from the aggregate (Fig. 8D). Aggregates that are $1-10 \mathrm{~mm}$ in size with excess densities in the range of $10^{-5}<\Delta \hat{\rho}<10^{-3}$ may need $1 \mathrm{~cm}$ to $1 \mathrm{~km}$ to release their entire initial solute content.

[28] The influence of excess density can be illustrated by considering mineral ballasting of marine aggregates. An aggregate of $a=5 \mathrm{~mm}$ in size with $\Delta \hat{\rho}=$ $10^{-5}$ would release all of its initial solute content at a depth of $3 \mathrm{~m}$ within $2.1 \mathrm{~h}$. An equally sized aggregate, incorporating mineral ballast, with $\Delta \hat{\rho}=10^{-3}$ would release its solute content within $1.8 \mathrm{~h}$ at a depth of $70 \mathrm{~m}$. Here, we exclude other processes such as microbial consumption and respiration, since, for example, the consumption rate of particulate organic carbon, which can be estimated as $0.004 \mathrm{~h}^{-1}$ (Ploug et al. 1999; Ploug 2001), is negligible over the course of $2 \mathrm{~h}$.

\section{Effect of a Constant Production}

[29] In addition to the processes considered above, we can account for degradation of organic matter within the aggregate. Several studies have shown a constant carbon-specific microbial degradation rate within the

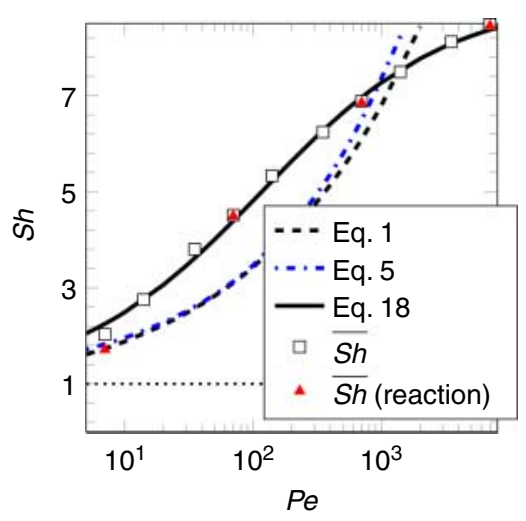

Fig. 7 Comparison of time-averaged, unsteady mass transfer enhancement $S h$ with the steady-state behavior as a function of $P e$ (in the absence of permeation, $D a \leq$ $10^{-4}$; the dotted line indicates $\left.S h=1\right)$. The red triangles represent the calculations taking into account a constant degradation rate inside the aggregates. The dashed, dot-dashed, and solid lines represent Eqs. 1, 5, and 18, respectively. 

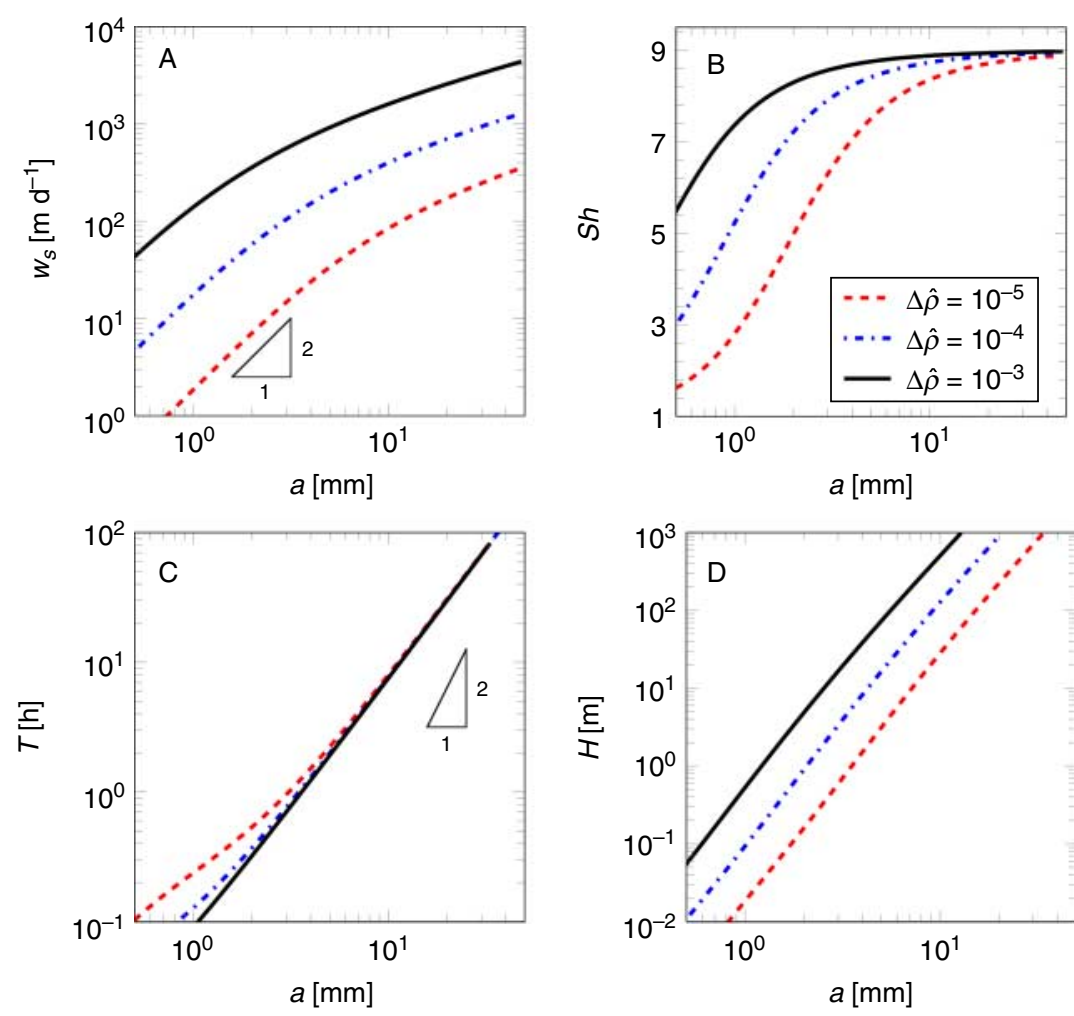

Fig. 8 Sinking velocity (A), Sherwood number (B), release time (C), and release depth (D) as a function of aggregate size $a$ for varying excess densities $\Delta \hat{\rho}$. The triangles denote the fitted power law. tion term in dimensionless form $(\tilde{R})$ will be

$$
\tilde{R}=\frac{R}{c_{0}} \frac{a^{2}}{D} \sim 1 .
$$

We define the release time as the time within which the concentration in the aggregate reaches $c^{\star}$, where $c^{\star}$ is when $\left(c_{0}-c^{\star}\right)\left(c_{0}-c_{\infty}\right)^{-1}=99 \%$, and $c_{\infty}$ is the steady-state concentration within the aggregate. We show $\overline{S h}$ as a function of $P e$ by including a constant production rate of $\tilde{R}=1$, denoted as symbols in Fig. 7. As shown in Fig. 7, there is only a slight change when a constant production was present.

\section{Significance to Aquatic Environments}

[30] Marine aggregates are considered the primary vehicles of downward transport of organic carbon in the ocean. The efficiency of the biological carbon pump is largely affected by the settling dynamics of marine aggregates. Furthermore, the latter directly controls elemental cycling and food web structures and constitutes a key parameter in the oceans' response to climate change. During the settling of marine aggregates, particulate organic carbon is gradually turned over by microbial respiration and solubilization, while solutes are constantly released into the water. This steady-state scenario is interrupted when the aggregate passes through density discontinuities (pycnocline). The excess dissolved organic matter will be released dynamically. In an attempt to quantify this dynamic process, we present a hydrodynamic study of the flow adjacent to and solute release from porous spheres. The results suggest a new relation for the enhanced solute release from the aggregate in terms of Sherwood number as a function of Peclét number. From this relation, release times and export depths can be readily obtained as a function of aggregate size and excess density. This small-scale particle dynamics is an important input for the global-scale biogeochemical models. 
Acknowledgments The authors thank M. H. Iversen for discussion and the three anonymous referees and the associate editor, G. Jackson, for their comments and suggestions. Thanks are also due to H. Marchant for going through the manuscript text.

\section{Appendix}

\section{Lattice Boltzmann Implementation}

[A1] Due to the axial symmetry of the problem, the three-dimensional flow field can be reduced to a quasi-two-dimensional description. Radial and axial velocity components and concentration fields were computed for spheres on an $r$ - $z$ grid (Fig. 1).

[A2] To solve Eqs. 11-14, we used a recently developed lattice-Boltzmann model (Guo et al. 2009; Zheng et al. 2010). The evolution equations for the velocity and concentration can be written as

$$
\begin{gathered}
f_{i}\left(\mathbf{x}+\mathbf{e}_{i} \delta_{t}, t+\delta t\right)-f_{i}(\mathbf{x}, t) \\
=-\frac{1}{\tau_{f}}\left[f_{i}(\mathbf{x}, t)-f_{i}^{(\mathrm{eq})}(\mathbf{x}, t)\right]+\mathbf{F}_{i}(x, t), \\
g_{i}\left(\mathbf{x}+\mathbf{e}_{i} \delta_{t}, t+\delta t\right)-g_{i}(\mathbf{x}, t)=-\frac{1}{\tau_{g}}\left[g_{i}(\mathbf{x}, t)-g_{i}^{(\mathrm{eq})}(\mathbf{x}, t)\right] \\
+\mathbf{G}_{i}(x, t),
\end{gathered}
$$

where $f_{i}(\mathbf{x}, t)$ and $g_{i}(\mathbf{x}, t)$ are the single-particle distribution function at position $\mathbf{x}$ and time $t$ along the direction represented by the subscript $i$ for fluid and density perturbation, respectively. The nondimensional relaxation times are defined by $\tau_{f}=v /\left(c_{s}^{2} \delta t\right)+1 / 2$ and $\tau_{g}=D /\left(c_{s}^{2} \delta t\right)+1 / 2$, where $c_{s}$ is the speed of sound (set as $c_{s}^{2}=1 / 3$ ) and $\delta t$ is the time increment (Succi 2001). According to the two-dimensional D2Q9 model (Qian et al. 1992), values of the variable $\mathbf{e}_{i}$ are discrete velocities defined as $\mathbf{e}_{i}=\left(\begin{array}{ll}e_{i n} & e_{i z}\end{array}\right), \quad i=$ $0,1,2, \ldots, 8$ and are specified as $\mathbf{e}_{0}=0, \quad \mathbf{e}_{1}=-\mathbf{e}_{3}=\left(\begin{array}{ll}1, & 0\end{array}\right), \quad \mathbf{e}_{2}=-\mathbf{e}_{4}=\left(\begin{array}{ll}0, & 1\end{array}\right)$, $\mathbf{e}_{5}=-\mathbf{e}_{7}=(1,1)$, and $\mathbf{e}_{6}=-\mathbf{e}_{8}=(-1,1)$. Furthermore, $f_{i}^{(\mathrm{eq})}(\mathbf{x}, t)$ and $g_{i}^{(\mathrm{eq})}(\mathbf{x}, t)$ are the equilibrium distribution functions

$$
f_{i}^{(\mathrm{eq})}=r \omega_{i} \rho\left[1+\frac{\mathbf{e}_{i} \cdot \mathbf{u}}{c_{s}^{2}}+\frac{\mathbf{u u}:\left(\mathbf{e}_{i} \mathbf{e}_{i}-c_{s}^{2} \mathbf{I}\right)}{2 \varepsilon c_{s}^{2}}\right]
$$

$$
g_{i}^{(\mathrm{eq})}=r \omega_{i} c\left[1+\frac{\mathbf{e}_{i} \cdot \mathbf{u}}{c_{s}^{2}}\right],
$$

where the weight $\omega_{i}$ is given by $\omega_{0}=4 / 9, \omega_{1 \sim 4}=1 / 9$, and $\omega_{5 \sim 8}=1 / 36$ and where $\mathbf{I}$ is the unit tensor. The symbols - and : denote the dot and dyadic products, respectively. Eqs. A3 and A4 also hold within the fluid region by requiring $\varepsilon=1$. The force terms $\mathbf{F}_{i}$ and $\mathbf{G}_{i}$ are given by

$$
\begin{gathered}
\mathbf{F}_{i}=\delta t\left(1-\frac{1}{2 \tau_{f}}\right) \frac{\left(\mathbf{e}_{i}-\mathbf{u}\right) \cdot \tilde{\alpha}}{c_{s}^{2}} f_{i}^{(\mathrm{eq})}, \\
\mathbf{G}_{i}=\delta t\left(1-\frac{1}{2 \tau_{g}}\right) \omega_{i} e_{i} \cdot \beta,
\end{gathered}
$$

with

$$
\begin{gathered}
\tilde{\alpha}_{z}=\alpha_{z}, \\
\tilde{\alpha}_{r}=\alpha_{r}+\frac{c_{s}^{2}}{r}\left[1-\frac{\left(2 \tau_{f}-1\right) u_{r}}{r}\right],
\end{gathered}
$$

where $\alpha=\left(\alpha_{n}, \alpha_{z}\right)=-(\varepsilon \nu / K) \mathbf{u}$ represents the Darcy force in porous media, which vanishes in the fluid domain. The additional term, $\beta=\left(\beta_{n} \beta_{z}\right)=(c, 0)$, is the same in both the fluid and the porous domain.

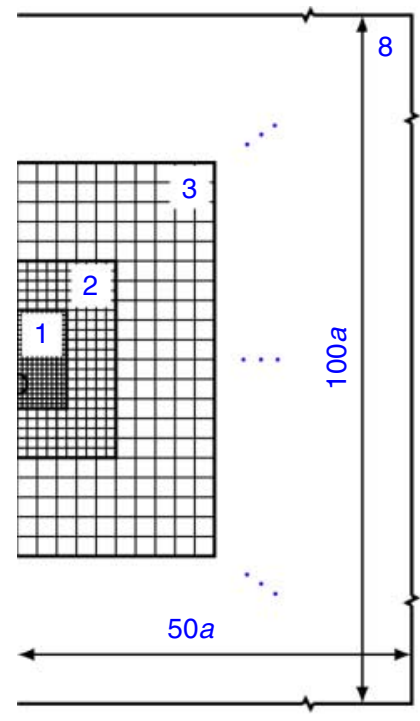

Fig. A1 The axisymmetric computational domain in the cylindrical coordinate with eight consecutive refinement levels $(1-8)$ and a total radial extent of $50 a$ in each direction. The aggregate is shown as a semicircle in the center of the figure. 
[A3] The hydrodynamic variables mass density $(\rho)$ and momentum $(\mathbf{j})$ together with concentration $(c)$ are computed by

$$
\begin{gathered}
\rho=\frac{1}{r} \sum_{i} f_{i}, \\
\mathbf{j}=\rho \mathbf{u}=\frac{\sum_{i} \mathbf{e}_{i} f_{i}}{\frac{r^{2}+\tau_{f} \delta t c_{s}^{2}}{r}+r \varepsilon \frac{\delta t}{2} \frac{\nu}{K}} \\
c=\frac{1}{r \varepsilon} \sum_{i} g_{i} .
\end{gathered}
$$

[A4] All simulations were conducted on rectangular $r-z$ domains of varying refinement levels. A grid refinement technique was applied with a hierarchy of eight nested grids with successive finer resolution toward the system center (Fig. A1). The spatial and temporal step sizes ( $\delta t$ and $\delta x$ ) of the finest grid were set to unity, which were successively doubled for the coarser grids. This choice ensured conservation of velocity $\mathbf{u}$ for all refinement levels. To restrain continuity of pressure, velocity, and their derivatives, as well as concentration at the interface between coarser and finer grids, the interpolation scheme of Liu and Khalili (2009) was used.

[A5] To calculate the reference drag, the aggregate has been taken as a solid sphere, at which all velocity components have been set to zero on the sphere surface (no-slip boundary conditions) (Guo and Zhao 2002). In the far field, the velocity was set to $w_{s}=$ constant, and the concentration of the solute was set to zero (Liu and Khalili 2008, 2009).

\section{References}

Adler, P. M. 1981. Streamlines in and around porous particles. J. Colloid Interface Sci. 81: 531-535, doi:10.1016/0021 -9797(81)90434-3.

Aidun, C. K., and J. R. Clausen. 2010. Lattice-Boltzmann method for complex flows. Annu. Rev. Fluid Mech. 42: 439-472, doi:10 .1146/annurev-fluid-121108-145519.

Alldredge, A. L., and C. C. Gotschalk. 1990. The relative contribution of marine snow of different origins to biological processes in coastal waters. Cont. Shelf Res. 10: 41-58, doi:10.1016/0278 -4343(90)90034-J.

Alldredge, A. L., and M. W. Silver. 1988. Characteristics, dynamics and significance of marine snow. Prog. Oceanogr. 20: 41-82, doi:10.1016/0079-6611(88)90053-5.
Asper, V. L., W. G. Deuser, G. A. Knauer, and S. E. Lohrenz. 1992. Rapid coupling of sinking particle fluxes between surface and deep ocean waters. Nature 357: 670-672, doi:10.1038/357670a0.

Basu, A. J., and A. Khalili. 1999. Computation of flow through fluidsediment interfaces in a benthic chamber. Phys. Fluids. 11: 1395-1405, doi:10.1063/1.870004.

Chen, S., and G. D. Doolen. 1998. Lattice Boltzmann method for fluid flows. Annu. Rev. Fluid Mech. 30: 329-364, doi:10.1146 /annurev.fluid.30.1.329.

Clift, A., J. R. Grace, and M. E. Weber. 1978. Bubbles, Drops and Particles. Academic Press.

Gorczyca, B., and J. Ganczarczyk. 2002. Flow rates through alum coagulation and activated sludge flocs. Water Qual. Res. J. Canada. 37: 389-398.

Guo, Z., H. Han, B. Shi, and C. Zheng. 2009. Theory of the lattice Boltzmann equation: Lattice Boltzmann model for ax symmetric flows. Phys. Rev. E Stat. Nonlin. Soft Matter Phys. 79: 046708, doi:10.1103/PhysRevE.79.046708.

Guo, Z., and T. S. Zhao. 2002. Lattice Boltzmann model for incompressible flows through porous media. Phys. Rev. E Stat. Nonlin. Soft Matter Phys. 66: 036304, doi:10.1103/PhysRevE.66 .036304 .

Iversen, M. H., and H. Ploug. 2010. Ballast minerals and the sinking carbon flux in the ocean: Carbon-specific respiration rates and sinking velocity of marine snow aggregates. Biogeosciences 7: 2613-2624, doi:10.5194/bg-7-2613-2010.

Karp-Boss, L., E. Boss, and P. Jumars. 1996. Nutrient fluxes to planktonic osmotrophs in the presence of fluid motion. Oceanogr. Mar. Biol. Annu. Rev. 34: 71-107.

Kindler, K., A. Khalili, and R. Stocker. 2010. Diffusion-limited retention of porous particles at density interfaces. Proc. Natl. Acad. Sci. USA. 107: 22163-22168, doi:10.1073/pnas.1012319108.

Kiørboe, T., H. Ploug, and U. H. Thygesen. 2001. Fluid motion and solute distribution around sinking aggregates. I. Small-scale fluxes and heterogeneity of nutrients in the pelagic environment. Mar. Ecol. Prog. Ser. 211: 1-13, doi:10.3354 /meps211001.

Leppard, G. G., D. Mavrocordatos, and D. Perret. 2004. Electronoptical characterization of nano- and micro-particles in raw and treated waters: An overview. Water Sci. Technol. 50: 1-8.

Li, X. Y., and B. E. Logan. 2001. Permeability of fractal aggregates. Water Res. 35: 3373-3380, doi:10.1016/S0043-1354(01) 00061-6.

Li, X. Y., Y. Yuan, and H. W. Wang. 2003. Hydrodynamics of biological aggregates of different sludge ages: An insight into the mass transport mechanisms of bioaggregates. Environ. Sci. Technol. 37: 292-299, doi:10.1021/es020764+.

Liu, B., and A. Khalili. 2008. Acceleration of steady-state lattice Boltzmann simulation for exterior flows. Phys. Rev. E Stat. Nonlin. Soft Matter Phys. 78: 056701, doi:10.1103/PhysRevE.78 .056701 . 
Liu, B., and A. Khalili. 2009. Lattice Boltzmann model for exterior flows with an annealing preconditioning method. Phys. Rev. E Stat. Nonlin. Soft Matter Phys. 79: 066701, doi:10.1103 /PhysRevE.79.066701.

Logan, B. E., and J. R. Hunt. 1987. Advantages to microbes of growth in permeable aggregates in marine systems. Limnol. Oceanogr. 32: 1034-1048, doi:10.4319/1o.1987.32.5.1034.

MacIntyre, S., A. L. Alldredge, and C. C. Gotschalk. 1995. Accumulation of marine snow at density discontinuities in the water column. Limnol. Oceanogr. 40: 449-468, doi:10.4319/lo.1995 .40.3.0449.

Masliyah, J. H., and M. Polikar. 1980. Terminal velocity of porous spheres. Can. J. Chem. Eng. 58: 299-302, doi:10.1002/cjce .5450580303 .

Nandakumar, K., and J. H. Masliyah. 1982. Laminar flow past a permeable sphere. Can. J. Chem. Eng. 60: 202-211, doi:10.1002 /cjce.5450600202.

Neale, G., N. Epstein, and W. Nader. 1973. Creeping flow relative to a permeable sphere. Chem. Eng. Sci. 28: 1865-1874, doi:10 .1016/0009-2509(73)85070-5.

Nield, D. A., and A. Bejan. 1998. Convection in Porous Media. Springer.

Ploug, H. 2001. Small-scale oxygen fluxes and remineralisation in sinking aggregates. Limnol. Oceanogr. 46: 1624-1631, doi:10.4319/lo.2001.46.7.1624.

Ploug, H., B. Adam, N. Musat, T. Kalvelage, G. Lavik, D. WolfGladrow, and M. M. M. Kuypers. 2011. Carbon, nitrogen and $\mathrm{O}_{2}$ fluxes associated with the cyanobacterium Nodularia spumigena in the Baltic Sea. ISME J. 5: 1549-1558, doi:10 .1038/ismej.2011.20.

Ploug, H., M. H. Iversen, and F. Gerhard. 2008. Ballast, sinking velocity, and apparent diffusivity within marine snow and fecal pellets: Implications for substrate turnover by attached bacteria. Limnol. Oceanogr. 53: 1878-1886, doi:10.4319/lo.2008 .53.5.1878.

Ploug, H., and U. Passow. 2007. Direct measurements of diffusivity within diatom aggregates containing transparent exopolymer particles. Limnol. Oceanogr. 52: 1-6, doi:10.4319/lo.2007.52 .1.0001.

Ploug, H., W. Stolte, E. H. G. Epping, and B. B. Jørgensen. 1999. Diffusive boundary layer, photosynthesis, and respiration of the colony-forming plankton algae, Phaeocystis sp. Limnol. Oceanogr. 44: 1949-1958, doi:10.4319/lo.1999.44.8.1949.

Qian, Y. H., D. D'Humières, and P. Lallemand. 1992. Lattice BGK models for Navier-Stokes equation. Europhys. Lett. 17: 479-484, doi:10.1209/0295-5075/17/6/001.

Rogak, S. N., and R. C. Flagan. 1990. Stokes drag on self-similar clusters of spheres. J. Colloid Interface Sci. 134: 206-218, doi:10.1016/0021-9797(90)90268-S.

Simon, M., H. P. Grossart, B. Schweitzer, and H. Ploug. 2002. Microbial ecology of organic aggregates in aquatic ecosystems. Aquat. Microb. Ecol. 28: 175-211, doi:10.3354/ame028175.

Succi, S. 2001. The Lattice Boltzmann Equation for Fluid Dynamics and Beyond. Oxford University Press.

Turner, J. T. 2002. Zooplankton fecal pellets, marine snow and sinking phytoplankton blooms. Aquat. Microb. Ecol. 27: 57-102, doi:10.3354/ame027057.

Vanni, M. 2000. Creeping flow over spherical permeable aggregates. Chem. Eng. Sci. 55: 685-698, doi:10.1016/S0009-2509(99) 00316-4.

White, F. M. 2005. Viscous Fluid Flow. McGraw-Hill.

Wolf-Gladrow, D. A. 1998. Lattice Gas Cellular Automata and Lattice Boltzmann Models: An Introduction. Springer.

Zheng, L., Z. Guo, B. Shi, and C. Zheng. 2010. Kinetic theory based lattice Boltzmann equation with viscous dissipation and pressure work for axisymmetric thermal flows. J. Comput. Phys. 229: 5843-5856, doi:10.1016/j.jcp.2010.04.026.

Received: 23 March 2012

Amended: 18 July 2012

Accepted: 1 October 2012 